\title{
CONVEX HULLS OF RANDOM WALKS
}

\author{
TIMOTHY LAW SNYDER AND J. MICHAEL STEELE
}

(Communicated by George C. Papanicolaou)

\begin{abstract}
Features related to the perimeter of the convex hull $C_{n}$ of a random walk in $\mathbb{R}^{2}$ are studied, with particular attention given to its length $L_{n}$. Bounds on the variance of $L_{n}$ are obtained to show that, for walks with drift, $L_{n}$ obeys a strong law. Exponential bounds on the tail probabilities of $L_{n}$ under special conditions are also obtained. We then develop simple expressions for the expected values of other features of $C_{n}$, including the number of faces, the sum of the lengths and squared lengths of the faces, and the number of faces of length $t$ or less.
\end{abstract}

\section{INTRODUCTION}

Let $Z_{j}, 1 \leq j<\infty$, be a sequence of independent, identically distributed random vectors with $Z_{j} \in \mathbb{R}^{2}, E Z_{j}=\mu$, and $E\left|Z_{j}\right|^{2}<\infty$. We set $S_{0}=0$ and let $S_{k}$ denote the partial sum of the $Z_{j}$ over $1 \leq j \leq k$. The purpose of this article is to investigate geometrical features of the convex hull $C_{n}$ of $\left\{S_{0}, S_{1}, \ldots, S_{n}\right\}$, and we are particularly concerned with $L_{n}$, the length of the perimeter of $C_{n}$.

The variable $L_{n}$ was first studied by Spitzer and Widom [11], and, by using a classical geometrical theorem of Cauchy (cf., Eggleston [6, p. 89]) that says the length of the perimeter of a convex set is the average of the length of its projections on a line through the origin, they found an elegant formula for the expectation

$$
E L_{n}=2 \sum_{k=1}^{n} \frac{1}{k} E\left|S_{k}\right|
$$

Spitzer and Widom's passage from Cauchy's formula to (1.1) called upon a combinatorial identity discovered earlier by Kac and proved by Dyson (cf., Kac [8]). A different proof of (1.1) was later given by Baxter [1], where the Cauchy representation was replaced by a purely combinatorial approach.

Received by the editors June 3, 1991.

1991 Mathematics Subject Classification. Primary 60D05, 60C05, 68U05, 62G99.

Key words and phrases. Strong laws, convex hulls, random walks, Efron-Stein Inequality, variance bounds, geometric probability.

The first author was supported in part by Georgetown University 1991 Summer Research Award and Georgetown University 1992 Junior Faculty Research Fellowship. The second author was supported in part by grants NSF DMS88-12868, NSA MDA904-89-H-2034, AFOSR-89-0301, and DAAL03-89-G-0092. 
In $\S 2$, we obtain more detailed information concerning the distribution of $L_{n}$. This is achieved by coupling the classical representation of Spitzer and Widom with the theory of jackknife inequalities. The end product is a general bound on the variance of $L_{n}$. When the $Z_{j}$ are assumed to have finite variance and nonzero mean, the variance bound can be used to obtain a law of large numbers for the length of the boundary of the convex hull of a random walk.

In $\S 3$ we obtain strong inequalities for the tail probabilities of $L_{n}$ when the $Z_{j}$ are assumed to be bounded; namely, we find that if $\left\|Z_{j}\right\|_{\infty} \leq 1$, then $P\left(\left|L_{n}-E L_{n}\right| \geq t\right) \leq 2 \exp \left(-t^{2} / 8 n \pi^{2}\right)$. The proof of this inequality rests on three ingredients: martingale differences, "change-one" random variables from the theory of jackknife inequalities, and a lemma of Hoeffding [7].

Section 4 provides a simple formula for the expected value of several quantities related to the faces of $C_{n}$. For example, for walks without drift, we obtain an intriguing identity for $E L_{n}^{(2)}$, the expected sum of the squared lengths of the faces

$$
E L_{n}^{(2)}=2 n\left(\sigma_{X}^{2}+\sigma_{Y}^{2}\right) .
$$

Here $\sigma_{X}^{2}$ and $\sigma_{Y}^{2}$ denote the variances of the coordinates of the summands $Z_{j}=\left(X_{j}, Y_{j}\right)$, and, curiously, $E L_{n}^{(2)}$ does not depend on the correlation of the coordinates.

The last section focuses on connections between the results of this paper and basic questions in the theory of algorithms. Brief note is made of some open problems.

\section{THE VARIANCE OF $L_{n}$}

In the course of their proof of the basic identity (1.1), Spitzer and Widom pointed out a useful representation of $L_{n}$, namely, that $L_{n}$ equals the average size of the numerical range of the projections of the set $\left\{S_{k}, 0 \leq k \leq n\right\}$ onto a line with direction $\theta$. Thus, if $p\left(S_{k}, \theta\right)$ denotes the projection of $S_{k}$ onto the line through the origin that makes an angle $\theta$ with the $x$-axis, then for all realizations of the random walk we have

$$
L_{n}=\int_{0}^{\pi}\left[\max _{0 \leq k \leq n} p\left(S_{k}, \theta\right)-\min _{0 \leq k \leq n} p\left(S_{k}, \theta\right)\right] d \theta,
$$

where $p\left(S_{k}, \theta\right)=X_{k} \cos \theta+Y_{k} \sin \theta$ for $S_{k}=\left(X_{k}, Y_{k}\right)$ and $0 \leq \theta<\pi$.

Because of jackknife inequalities like that of Efron and Stein [5], this representation of Spitzer and Widom suggests a convenient way to bound the variance of $L_{n}$. Although the original Efron-Stein Inequality applies only to functions of random variables $S\left(X_{1}, X_{1}, \ldots, X_{n}\right)$ that are almost surely invariant under permutations of their arguments, a modified version given in Steele [12] can be applied to any real-valued function $Y\left(X_{1}, X_{2}, \ldots, X_{n}\right)$ of $n$ independent identically distributed random vectors $X_{i}$. Specifically, if we write $Y=$ $Y\left(X_{1}, X_{2}, \ldots, X_{n}\right)$ and let $Y^{(j)}=Y\left(X_{1}, X_{2}, \ldots, X_{j-1}, \widetilde{X}_{j}, X_{j+1}, \ldots, X_{n}\right)$, where the $2 n$ random vectors $X_{j}$ and $\tilde{X}_{j}$, for $1 \leq i \leq n$, are i.i.d., then Steele [12] shows that

$$
\operatorname{Var} Y \leq \frac{1}{2} E \sum_{j=1}^{n}\left(Y-Y^{(j)}\right)^{2}
$$


Applying (2.2) to $L_{n}$ viewed as a (non-permutation-invariant) function of the $Z_{j}$, we obtain

$$
\operatorname{Var} L_{n} \leq \frac{1}{2} E \sum_{j=1}^{n}\left(L_{n}-L_{n}^{(j)}\right)^{2}
$$

where $L_{n}^{(j)}$ denotes the length of the convex hull of $\left\{S_{1}^{(j)}, S_{2}^{(j)}, \ldots, S_{n}^{(j)}\right\}$ and the $S_{k}^{(j)}$ are formed by replacing the $j$ th summand $Z_{j}$ by the independent copy $\widetilde{Z}_{j}$.

To bound $E\left(L_{n}-L_{n}^{(j)}\right)^{2}$, we use the parameterized range function

$$
R(n, \theta)=\max _{1 \leq k \leq n} p\left(S_{k}, \theta\right)-\min _{1 \leq k \leq n} p\left(S_{k}, \theta\right),
$$

so the Spitzer and Widom representation (2.1) becomes $L_{n}=\int_{0}^{\pi} R(n, \theta) d \theta$. We can now write

$$
E\left(L_{n}-L_{n}^{(j)}\right)^{2}=E\left\{\int_{0}^{\pi}\left(R(n, \theta)-R^{(j)}(n, \theta)\right) d \theta\right\}^{2}
$$

where $R^{(j)}(n, \theta)$ is just $R(n, \theta)$ with $S_{k}$ replaced by $S_{k}^{(j)}$ for each $1 \leq k \leq n$. Our first lemma bounds the change in the range function in terms of $Z_{j}$ and its redrawn counterpart $\tilde{Z}_{j}$.

Lemma 2.1. For all $1 \leq j \leq n$,

$$
\left|R(n, \theta)-R^{(j)}(n, \theta)\right| \leq\left|p\left(Z_{j}, \theta\right)-p\left(\tilde{Z}_{j}, \theta\right)\right| .
$$

Proof. Consider the effect on $p\left(S_{k}, \theta\right)$ when $Z_{j}$ is replaced with $\widetilde{Z}_{j}$. If $j>k$, then $p\left(S_{k}, \theta\right)=p\left(S_{k}^{(j)}, \theta\right)$. If $j \leq k$, then $p\left(S_{k}, \theta\right)=p\left(S_{k}^{(j)}, \theta\right)+p\left(Z_{j}, \theta\right)-$ $p\left(\widetilde{Z}_{j}, \theta\right)$. Hence, for all $j$,

$$
p\left(S_{k}, \theta\right) \leq p\left(S_{k}^{(j)}, \theta\right)+\left[p\left(Z_{j}, \theta\right)-p\left(\widetilde{Z}_{j}, \theta\right)\right]_{+},
$$

where we write $[x]_{+}=x$ if $x \geq 0$ and $[x]_{+}=0$ if $x<0$ (and $[x]_{-}=0$ if $x \geq 0$ and $[x]_{-}=x$ if $\left.x<0\right)$. We therefore have that

$$
\max _{1 \leq k \leq n} p\left(S_{k}, \theta\right) \leq \max _{1 \leq k \leq n} p\left(S_{k}^{(j)}, \theta\right)+\left[p\left(Z_{j}, \theta\right)-p\left(\tilde{Z}_{j}, \theta\right)\right]_{+} \cdot
$$

Similarly, we find

$$
\min _{1 \leq k \leq n} p\left(S_{k}, \theta\right) \geq \min _{1 \leq k \leq n} p\left(S_{k}^{(j)}, \theta\right)+\left[p\left(Z_{j}, \theta\right)-p\left(\tilde{Z}_{j}, \theta\right)\right]_{-},
$$

so, combining (2.8) and (2.9), one has

(2.10) $R(n, \theta)-R^{(j)}(n, \theta) \leq\left[p\left(Z_{j}, \theta\right)-p\left(\widetilde{Z}_{j}, \theta\right)\right]_{+}-\left[p\left(Z_{j}, \theta\right)-p\left(\widetilde{Z}_{j}, \theta\right)\right]_{-}$;

but, by the identity $[x]_{+}-[x]_{-}=|x|$ and by the symmetry of $R(n, \theta)$ and $R^{(j)}(n, \theta)$, we can replace the left side of $(2.10)$ with the corresponding absolute value, thus completing the lemma.

If we now insert the bound of Lemma 2.1 into equation (2.5) and apply the jackknife bound (2.3), we obtain

$$
\operatorname{Var} L_{n} \leq \frac{1}{2} E \sum_{j=1}^{n}\left[\int_{0}^{\pi}\left|p\left(Z_{j}, \theta\right)-p\left(\tilde{Z}_{j}, \theta\right)\right| d \theta\right]^{2} .
$$

To bound (2.11), write $Z_{j}=\left(X_{j}, Y_{j}\right)$, and let $\sigma_{X}^{2}$ and $\sigma_{Y}^{2}$ be the variances of the $X_{j}$ and $Y_{j}$, respectively. 
Lemma 2.2. For all $1 \leq j \leq n$,

$$
E\left[\int_{0}^{\pi}\left|p\left(Z_{j}, \theta\right)-p\left(\tilde{Z}_{j}, \theta\right)\right| d \theta\right]^{2} \leq \pi^{2}\left(\sigma_{X}^{2}+\sigma_{Y}^{2}\right) .
$$

Proof. We first apply the Schwarz Inequality to the left-hand side of (2.12) to get

$$
E\left[\int_{0}^{\pi}\left|p\left(Z_{j}, \theta\right)-p\left(\tilde{Z}_{j}, \theta\right)\right| d \theta\right]^{2} \leq \pi E \int_{0}^{\pi}\left|p\left(Z_{j}, \theta\right)-p\left(\tilde{Z}_{j}, \theta\right)\right|^{2} d \theta
$$

Since $Z_{j}$ and $\widetilde{Z}_{j}$ are identically distributed, by the definition of $p\left(Z_{j}, \theta\right)$ one has

$$
\begin{aligned}
E\left|p\left(Z_{j}, \theta\right)-p\left(\widetilde{Z}_{j}, \theta\right)\right|^{2} & =2 \operatorname{Var}\left[p\left(Z_{j}, \theta\right)\right] \\
& =2\left(\sigma_{X}^{2} \cos ^{2} \theta+\sigma_{Y}^{2} \sin ^{2} \theta+2 \cos \theta \sin \theta \rho_{X Y} \sigma_{X} \sigma_{Y}\right),
\end{aligned}
$$

so

$E \int_{0}^{\pi}\left|p\left(Z_{j}, \theta\right)-p\left(\tilde{Z}_{j}, \theta\right)\right|^{2} d \theta=2\left(\sigma_{X}^{2} \int_{0}^{\pi} \cos ^{2} \theta d \theta+\sigma_{Y}^{2} \int_{0}^{\pi} \sin ^{2} \theta d \theta\right)$

$$
\begin{aligned}
& +4 \rho_{X Y} \sigma_{X} \sigma_{Y} \int_{0}^{\pi} \cos \theta \sin \theta d \theta \\
= & \pi\left(\sigma_{X}^{2}+\sigma_{Y}^{2}\right) .
\end{aligned}
$$

This proves the lemma.

Lemma 2.2 and the jackknife inequality (2.11) provide a basic bound on the variance of $L_{n}$.

Theorem 2.3. For all $n \geq 1$,

$$
\operatorname{Var} L_{n} \leq \pi^{2} n\left(\sigma_{X}^{2}+\sigma_{Y}^{2}\right) / 2 .
$$

The $O(n)$ bound can be used in a familiar way with an interpolation argument and the Borel-Cantelli Lemma to give a strong law for $L_{n}$ when $\mu \neq 0$. To sketch the argument, we first need to check that

$$
E L_{n} \sim 2 n|\mu| \text {. }
$$

This follows from the Spitzer and Widom identity (1.1) if $E\left|S_{k}\right| \sim k|\mu|$, but, for summands with finite variance, the latter relation is well known and can be extracted using, for example, a theorem of Marcinkiewicz and Zygmund (see Chow and Teicher $[4$, p. 125]).

Finally, by taking $n_{k}=k^{2}$ and $C=\pi^{2}\left(\sigma_{X}^{2}+\sigma_{Y}^{2}\right) / 2$ and applying our variance bound of $C n_{k}$ with Chebyshev's inequality, we find for any $\varepsilon>0$ that

$$
\sum_{k=1}^{\infty} P\left(\left(L_{n_{k}}-E L_{n_{k}}\right) / n_{k} \geq \varepsilon\right) \leq \frac{C}{2 \varepsilon^{2}} \sum_{k=1}^{\infty} \frac{1}{k^{2}}<\infty .
$$

We therefore see that $\left(L_{n_{k+1}}-E L_{n_{k}}\right) / n_{k} \rightarrow 0$ almost surely as $k \rightarrow \infty$. Since $E L_{n}$ is monotone, there is no difficulty in completing the interpolation argument needed to show that $L_{n} / n$ converges almost surely.

\section{LARGE DEVIATIONS}

In this section we assume that the steps of the random walk are bounded. We use the following bound on the moment-generating function of $L_{n}$ to provide bounds on the tail probabilities of $L_{n}-E L_{n}$. 
Lemma 3.1. If $\left\|Z_{j}\right\|_{\infty} \leq 1$, then for all $t \geq 0$

$$
E\left[\exp \left(t\left(L_{n}-E L_{n}\right)\right)\right] \leq \exp \left(2 n \pi^{2} t^{2}\right) .
$$

Before proving Lemma 3.1, we note that applying the lemma twice with Markov's Inequality gives us a basic tail bound.

Corollary 3.2. If $\left\|Z_{j}\right\|_{\infty} \leq 1$, then for all $t \geq 0$

$$
P\left(\left|L_{n}-E L_{n}\right| \geq t\right) \leq 2 \exp \left(-t^{2} / 8 n \pi^{2}\right) .
$$

Proof. We first note that the Spitzer-Widom representation and Jensen's Inequality yield

$$
\begin{aligned}
\exp \left(\pi^{-1} t\left(L_{n}-E L_{n}\right)\right) & =\exp \left(\frac{t}{\pi} \int_{0}^{\pi}(R(n, \theta)-E R(n, \theta)) d \theta\right) \\
& \leq \frac{1}{\pi} \int_{0}^{\pi} \exp (t(R(n, \theta)-E R(n, \theta))) d \theta .
\end{aligned}
$$

Now if $\mathscr{F}_{j}$ denotes the $\sigma$-field generated by $\left\{Z_{1}, Z_{2}, \ldots, Z_{j}\right\}$ and $\mathscr{F}_{0}$ denotes the trivial $\sigma$-field, then we have for each $\theta$ that

$$
R(n, \theta)-E R(n, \theta)=\sum_{j=1}^{n} d_{j}(\theta),
$$

where $d_{j}$ is the martingale difference defined by

$$
d_{j}(\theta)=E\left[R(n, \theta) \mid \mathscr{F}_{j}\right]-E\left[R(n, \theta) \mid \mathscr{F}_{j-1}\right]
$$

for $1 \leq j \leq n$. As in $\S 2$, we use the "change-one" variables $R^{(j)}(n, \theta)$ and note that since $\tilde{Z}_{j}$ is independent of $\mathscr{F}_{j}$, we have

$$
E\left[R^{(j)}(n, \theta) \mid \mathscr{F}_{j}\right]=E\left[R(n, \theta) \mid \mathscr{F}_{j-1}\right] ;
$$

thus, we can write $d_{j}$ conveniently as

$$
d_{j}(\theta)=E\left[R(n, \theta)-R^{(j)}(n, \theta) \mid \mathscr{F}_{j}\right] .
$$

By Lemma 2.1 and the bound $\left\|Z_{j}\right\|_{\infty} \leq 1$, we therefore find that $\left\|d_{j}(\theta)\right\|_{\infty} \leq 2$ for all $\theta$.

Next we note that

$$
E\left[\exp \left(t \sum_{j=1}^{n} d_{j}(\theta)\right)\right]=E\left[\exp \left(t \sum_{j=1}^{n-1} d_{j}(\theta)\right) E\left[\exp \left(t d_{n}(\theta)\right) \mid \mathscr{F}_{n-1}\right]\right] .
$$

By a lemma of Hoeffding [7, Equation 4.1], if $X$ has mean zero and if $\|X\|_{\infty} \leq$ $a$, then $E[\exp (t X)] \leq \exp \left(a^{2} t^{2} / 2\right)$. Applying Hoeffding's lemma to the expectation of $d_{n}(\theta)$ in $(3.8)$, we see that

$$
E\left[\exp \left(t \sum_{j=1}^{n} d_{j}(\theta)\right)\right] \leq \exp \left(2 t^{2}\right) E\left[\exp \left(t \sum_{j=1}^{n-1} d_{j}(\theta)\right)\right] .
$$

Repeating the argument for $n-1, n-2, \ldots$, we find for all $\theta$ that

$$
E\left[\exp \left(t \sum_{j=1}^{n} d_{j}(\theta)\right)\right] \leq \exp \left(2 n t^{2}\right)
$$

so on returning to (3.3), we can apply (3.10) to obtain (3.1). 


\section{FunCtions OF THE FACE LENGTHS}

We will now give a formula that generalizes that of Spitzer and Widom and provides new information on the expectations of several other features of the convex hull of a random walk. The derivation of these expectations will depend on the following

Lemma (Baxter [1]). Let $\left\{z_{1}, z_{2}, \ldots, z_{n}\right\}$ be vectors in $\mathbb{R}^{2}$ such that all $2^{n}$ subsets of $\left\{z_{1}, z_{2}, \ldots, z_{n}\right\}$ have distinct sums. For any permutation $\sigma$ of $1,2, \ldots, n$, let $s_{k}(\sigma)=\sum_{i=1}^{k} z_{\sigma(i)}$ for $1 \leq k \leq n$, and let $s_{0}=0$. Then, for any nonempty m-set $A \subset\{1,2, \ldots, n\}$, the vector $z_{A}=\sum_{j \in A} z_{j}$ is a face of the convex hull $c_{n}(\sigma)$ of $\left\{s_{0}(\sigma), s_{1}(\sigma), \ldots, s_{n}(\sigma)\right\}$ for exactly

$$
2(m-1) !(n-m) !
$$

of the permutations of $\{1,2, \ldots, n\}$.

For any $f: \mathbb{R} \rightarrow \mathbb{R}$, let

$$
G_{n}=\sum_{i=1}^{H_{n}} f\left(\left|e_{i}\right|\right),
$$

where $\left|e_{1}\right|,\left|e_{2}\right|, \ldots,\left|e_{H_{n}}\right|$ are the lengths of the faces of the convex hull of $\left\{S_{0}, S_{1}, \ldots, S_{n}\right\}$. Here one should note that $H_{n}$ is a random variable. If we suppose that the $Z_{j}$ for $1 \leq j \leq n$ have a common density, then with probability one $\left\{Z_{j}, 1 \leq j \leq n\right\}$ is a set with distinct subset sums, and we can apply Baxter's Lemma. If we let $G_{n}(\sigma)=G_{n}\left(Z_{\sigma(1)}, Z_{\sigma(2)}, \ldots, Z_{\sigma(n)}\right)$, then we can now determine the sum of $G_{n}(\sigma)$ over all $\sigma$ in terms of a sum over all nonempty $A \subset\{1,2, \ldots, n\}$. Let $C_{n}(\sigma)$ denote the convex hull of $S_{0}$ and $S_{k}(\sigma)=Z_{\sigma(1)}+\cdots+Z_{\sigma(k)}$, where $1 \leq k \leq n$. Letting $Z_{A}$ denote $\sum_{j \in A} Z_{j}$ and 1 the standard indicator function, we have

$$
\begin{aligned}
\sum_{\sigma} G_{n}(\sigma) & =\sum_{\sigma} \sum_{A} f\left(\left|Z_{A}\right|\right) \mathbf{1}\left(Z_{A} \text { is a face of } C_{n}(\sigma)\right) \\
& =\sum_{A} f\left(\left|Z_{A}\right|\right) \sum_{\sigma} \mathbf{1}\left(Z_{A} \text { is a face of } C_{n}(\sigma)\right) \\
& =\sum_{A} f\left(\left|Z_{A}\right|\right) 2(|A|-1) !(n-|A|) !
\end{aligned}
$$

where the last step follows from Baxter's Lemma. The left side of (4.3) has expectation $n ! E G_{n}$, so, using the fact that $E f\left(\left|Z_{A}\right|\right)=E f\left(\left|S_{k}\right|\right)$ if $|A|=k$, we see that

$$
n ! E G(n)=2 \sum_{k=1}^{n}\left(\begin{array}{l}
n \\
k
\end{array}\right) E f\left(\left|S_{k}\right|\right)(k-1) !(n-k) ! .
$$

This yields the following lemma.

Lemma 4.1. If the summands $Z_{j}$, where $1 \leq j \leq k$, have a common density, then, for any $f$ for which $E f\left(\left|S_{k}\right|\right)$ exists,

$$
E G_{n}=2 \sum_{k=1}^{n} \frac{E f\left(\left|S_{k}\right|\right)}{k}
$$


The identity (4.5) turns out to be quite powerful. For example, when $f(x)=$ 1 , then $G_{n}=H_{n}$, and we recapture the interesting relation

$$
E H_{n}=2 \sum_{k=1}^{n} \frac{1}{k} \sim 2 \ln n,
$$

which is due to Baxter [1]. Further, if $f(x)=x$, then (4.5) returns to us the formula of Spitzer and Widom, (1.1), which was originally proved via Cauchy's formula, but here emerges from purely combinatorial calculations.

These two choices of $f$ are not the only ones for which interesting formulas result. If we consider $f(x)=x^{2}$ and define $L_{n}^{(2)}$ to be the sum of the squared edge lengths of $C_{n}$, then we find for $Z_{j}=\left(X_{j}, Y_{j}\right)$ that

$$
\left|S_{k}\right|^{2}=\left(X_{1}+X_{2}+\cdots+X_{k}\right)^{2}+\left(Y_{1}+Y_{2}+\cdots+Y_{k}\right)^{2} \text {. }
$$

If we take the $X_{j}$ and $Y_{j}$ to be distributed with mean zero and variance $\sigma_{X}^{2}$ and $\sigma_{Y}^{2}$, respectively, then $E\left|S_{k}\right|^{2}=k\left(\sigma_{X}^{2}+\sigma_{Y}^{2}\right)$, so

$$
E\left[\sum_{k=1}^{n} \frac{\left|S_{k}\right|^{2}}{k}\right]=2 \sum_{k=1}^{n}\left(\sigma_{X}^{2}+\sigma_{Y}^{2}\right)=2 n\left(\sigma_{X}^{2}+\sigma_{Y}^{2}\right),
$$

from which we find the remarkable formula

$$
E L_{n}^{(2)}=2 n\left(\sigma_{X}^{2}+\sigma_{Y}^{2}\right)
$$

that is, the expected sum of squares of the faces of the convex hull of a random walk is just $n$ times twice the variance of an individual step. Although the development of (4.8) has proceeded somewhat seamlessly, it might otherwise have been difficult to guess or prove (4.8) without Lemma 4.1.

The lemma can also be used to determine the expected cardinality of subsets of the faces that satisfy certain properties. As an example, we determine the expected number of hull faces that are of length $t$ or less under a normality assumption.

Proposition. If $Z_{j}=\left(X_{j}, Y_{j}\right)$ are i.i.d. $N\left(0, \sigma^{2} I\right)$, then one has an exact expression for the number of faces $e_{i}$ of $C_{n}$ having length $t$ or less:

$$
E\left|\left\{i:\left|e_{i}\right| \leq t\right\}\right|=2 \sum_{k=1}^{n} \frac{1}{k}\left(1-e^{-t^{2} / 2 k \sigma^{2}}\right) .
$$

Proof. Let $f(x)=\mathbf{1}(x \leq t)$. To prove (4.9), we use Lemma 4.1:

$$
\begin{aligned}
E G_{n} & =2 \sum_{k=1}^{n} \frac{P\left(\left|S_{k}\right| \leq t\right)}{k} \\
& =2 \sum_{k=1}^{n} \frac{1}{k} P\left(\left(\sum_{j=1}^{k} X_{j}\right)^{2}+\left(\sum_{j=1}^{k} Y_{j}\right)^{2} \leq t^{2}\right) .
\end{aligned}
$$

Since the $X_{j}$ and $Y_{j}$ are normally distributed, a traditional calculation tells us that $\left(\sum_{j=1}^{k} X_{j}\right)^{2}+\left(\sum_{j=1}^{k} Y_{j}\right)^{2}$ is exponentially distributed with mean $1 / \lambda=$ $2 k \sigma^{2}$. We see then that $P\left(\left|S_{k}\right| \leq t\right)=1-e^{-t^{2} / 2 k \sigma^{2}}$, and thus (4.9) follows immediately. 


\section{CONCLUDING REMARKS}

A basic topic in computational geometry is the determination of the convex hull of point sets in $\mathbb{R}^{2}$. One result of particular interest is the "output sensitive" algorithm of Kirkpatrick and Seidel [9] that determines the convex hull of an $n$ set of $\mathbb{R}^{2}$ in time $O(n \log h)$, where $h$ is the number of faces of the convex hull. The Kirkpatrick-Seidel algorithm asymptotically outperforms older $O(n \log n)$ algorithms when a point set is such that the number of faces of its convex hull is relatively small.

This situation prevails in some, but not all, probabilistic contexts. For example, Rényi and Sulanke [10] showed that for points selected uniformly from the unit square, the expected number of hull faces is $E h \sim(8 / 3) \ln n$. This implies that a convex hull for such point sets can be found in $O(n \log \log n)$ expected time by the Kirkpatrick-Seidel algorithm. On the other hand, for variables that are distributed uniformly in the unit disk, Rényi and Sulanke [10] showed that $E h \sim \alpha n^{1 / 3}$, where $\alpha$ is constant. Thus, for these point sets, we can expect the Kirkpatrick-Seidel algorithm to run in time that is the same order of magnitude as that required by older methods.

One of the motivations for studying the convex hull of random walks is that it provides a second rich stochastic context in which the Kirkpatrick-Seidel algorithm leads to an order of magnitude expected speedup in the time needed to determine the convex hull. Specifically, the results of $\S 4$ imply that an $O(n \log \log n)$ expected running time suffices to determine the convex hull of a random walk. Though there exist algorithms that run in linear expected time (see, e.g., Bentley and Shamos [2] and Borgwardt, Gaffke, Jünger, and Reinelt [3]), these algorithms still exhibit $O(n \log n)$ worst-case behavior.

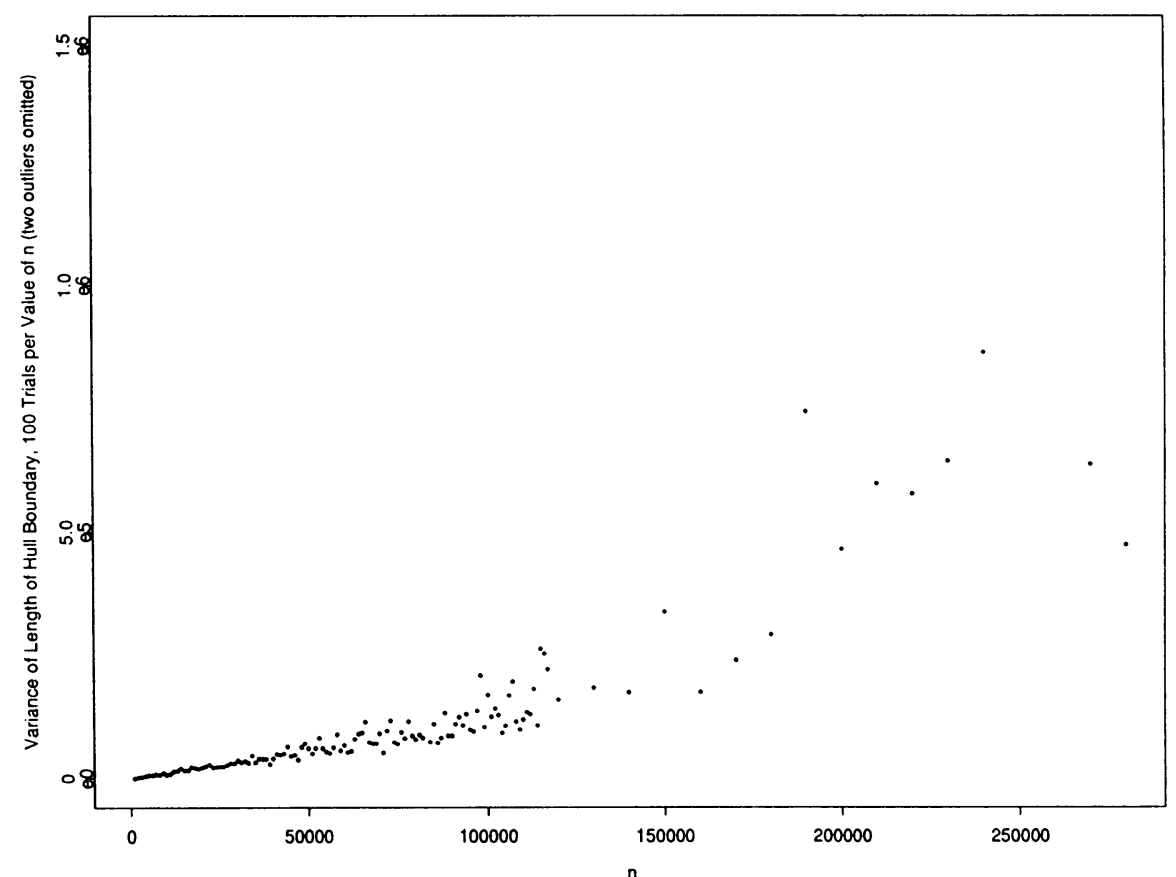

FIGURE 1 
There are several natural and concrete problems that the methods of this article leave unresolved. First, the variance bound of $O(n)$ provided by the jackknife inequality appears to be difficult to improve, but there is no compelling reason to expect that $O(n)$ is the correct order of magnitude; Var $L_{n}$ may indeed be smaller. One would expect that an $o(n)$ bound would contribute to the understanding of the behavior of $L_{n}$ in the zero-drift case. Results from simulations, however, as illustrated in Figure 1, indicate that an $o(n)$ bound in the zero-drift case may not hold. In a complementary direction, the analysis of $\S 4$ suggests that a distributional limit result, perhaps even a Poisson law, might hold for the number of edges of length less than $t$; however, the main tools used here, the Spitzer-Widom representation and Baxter's Lemma, do not seem to offer progress on either of these problems.

\section{REFERENCES}

1. G. Baxter, A combinatorial lemma for complex numbers, Ann. Math. Statist. 32 (1961), 901-904.

2. J. L. Bentley and M. I. Shamos, Divide and conquer for linear expected time, Inform. Process. Lett. 7 (1978), 87-91.

3. K. H. Borgwardt, N. Gaffke, M. Jünger, and G. Reinelt, Computing the convex hull in the Euclidean plane in linear expected time, DIMACS Series in Discrete Mathematics and Theoretical Computer Science, vol. 4, Amer. Math. Soc., Providence, RI, 1991, pp. 91-106.

4. Y. S. Chow and H. Teicher, Probability theory: Independence, interchangeability, martingales, Springer-Verlag, New York, 1988.

5. B. Efron and C. Stein, The jackknife estimate of variance, Ann. Statist. 10 (1981), 586-596.

6. H. G. Eggleston, Convexity, Cambridge Univ. Press, London, 1958.

7. W. Hoeffding, Probability inequalities for sums of bounded random variables, J. Amer. Statist. Assoc. 58 (1963), 13-30.

8. M. Kac, Toeplitz matrices, translation kernels, and a related problem in probability theory, Duke Math. J. 21 (1954), 501-509.

9. D. G. Kirkpatrick and R. Seidel, The ultimate convex hull algorithm?, SIAM J. Comput. 15 (1986), 89-96.

10. A. Rényi and R. Sulanke, Über die konvexe Hülle von $n$ zufällig gewählten Punkten, $\mathrm{Z}$. Wahrsch. Verw. Gebiete 2 (1963), 75-84.

11. F. Spitzer and H. Widom, The circumference of a convex polygon, Proc. Amer. Math. Soc. 12 (1961), 506-509.

12. J. M. Steele, An Efron-Stein inequality for non-symmetric statistics, Ann. Statist. 14 (1986), 753-758.

Department of Computer Science, Georgetown University, Washington, District of COLUMBIA 20057

E-mail address: tim@normal.georgetown.edu

Department of Statistics, The Wharton School, University of Pennsylvania, PhilaDElPhia, PENNSYlVANia 19104

E-mail address: steele@wharton.upenn.edu 